\title{
Clinical Outcomes and Prognosis of Small-Cell Lung Cancer(SCLC) Patients After Surgery: a Single- Centre Retrospective Cohort Study from Peking Union Medical College Hospital
}

\section{Yeye Chen}

Peking Union Medical College Hospital https://orcid.org/0000-0001-6654-7701

Lei Liu

Peking Union Medical College Hospital

Cheng Huang

Peking Union Medical College Hospital

Zhenhuan Tian

Peking Union Medical College Hospital

Hongsheng Liu

Peking Union Medical College Hospital

Shanqing LI (D Isq6768@sohu.com )

Research article

Keywords: small-cell lung cancer, surgical treatment, prognosis analysis

Posted Date: July 16th, 2020

DOI: https://doi.org/10.21203/rs.3.rs-42544/v1

License: (a) (i) This work is licensed under a Creative Commons Attribution 4.0 International License. Read Full License 


\section{Abstract}

Background: Small-cell lung cancer (SCLC) patients are generally not considered to be candidates for surgical intervention. The role of surgery in the multidisciplinary treatment of SCLC remains controversial. The study aims to summarize and analyse the clinical characteristics and prognosis of SCLC patients after surgical treatment and identify the related prognostic factors.

Methods: The clinical data of 130 SCLC patients (99 males and 31 females) who were treated by surgery and confirmed by postoperative pathological examination in Peking Union Medical College Hospital from April 2004 to April 2019 were analysed retrospectively.

Results: The median age was 60 years (30-83 years), and the median disease duration was 2 months (0.5-48 months). The patients did not have specific clinical or imaging manifestations. Postoperative pathological examination revealed that 36 patients had stage I, 22 patients had stage II, 65 patients had stage III, and seven patients had stage IV SCLC. The median overall survival time was 50 months (95\% confidence interval, 10.8-89.2 months). The median survival time of stage I, II, III, and IV SCLC patients were 148 months, 42 months, 32 months, and 10 months, respectively. In patients who underwent surgical treatment, postoperative adjuvant therapy and tumour stage were two significant influencing factors of survival (Cox multivariate analysis $P<0.05$ ).

Conclusions: Surgical treatment combined with adjuvant therapy can achieve satisfactory outcomes in selected SCLC patients. Adjuvant therapy and tumour stage are independent risk factors. Surgical treatment is indicated in SCLC patients preoperatively staged as I-IIla, and anatomical lobectomy and lymphadenectomy combined with postoperative adjuvant therapy are recommended.

\section{Background}

Small-cell lung cancer (SCLC), which accounts for approximately $13-15 \%$ of all primary lung malignancies, is highly aggresive and extremely difficult to treat [1]. The median survival time of untreated SCLC patients is only 2-4 months, and more than two-thirds of patients have distant metastasis at the time of diagnosis [2,3]. At present, surgery remains an auxiliary approach in the treatment of SCLC, being recommended for only early SCLC patients with negative lymph nodes in the guidelines provided by the National Comprehensive Cancer Network (NCCN) [4]. However, with advances in diagnostic and surgical technologies, many researchers have begun to challenge the existing view regarding the value of surgery in treating SCLC and have attempted to expand the surgical indications of and re-evaluate the role of surgery in SCLC treatment.

In the present study, the data of SCLC patients who underwent surgery-centred comprehensive treatment in one centre, Peking Union Medical College Hospital (PUMCH), were retrospectively analysed. We explored the value of surgery in treating SCLC and identified the relevant prognostic factors in the hopes of clarifying the significance of surgery in the prognosis of SCLC patients and reassessing its value in the multi-disciplinary treatment (MDT) of SCLC. 


\section{Methods}

\subsection{Study subjects and clinical indicators}

This study enrolled SCLC patients who underwent surgical treatment in PUMCH from April 2004 to April 2019. The diagnosis of SCLC was confirmed by postoperative pathological examination in all included patients. General data, including clinical characteristics, surgery, pathological stage, and auxiliary treatment, were summarized. Follow-up was conducted at the outpatient clinic or by telephone. Patients in stable condition were re-examined every three months during the first 1-2 years after surgery, every 6 months during the 3rd to 5th years, and thereafter once every year for 5 years and more. Patients with tumour recurrence were re-examined and treated immediately according to the progression of the disease. The latest follow-up data included in the study were from April 2020. The overall survival(OS) was from the date of surgery to the date of death in deceased patients. For the patients who survived and the patiens who were lost to follow-up, OS was defined as the time from the date of surgery to the date of the last follow-up.

\subsection{Surgery-centred comprehensive treatment}

All patients received surgery-centred comprehensive treatment. Subpulmonary lobectomy, lobectomy, compound lobectomy, or pneumonectomy was performed by either posterolateral thoracotomy (PLT) or video-assisted thoracic surgery (VATS), according to the location and size of the tumour, as well as the preoperative biopsy or intraoperative frozen pathology results. The SCLC was staged based on the 7th edition of the American Joint Committee On Cancer (AJCC) Cancer Staging Manual [5]. Patients were examined through bronchoscopy- or computed tomography (CT)-guided puncture for pathological diagnosis before surgery according to their clinical situation, and a portion of patients who were diagnosed pathologically were given adjuvant chemotherapy or a combination of chemotherapy and radiotherapy before surgery. Postoperative adjuvant chemotherapy was given according to the patient's pathological condition, and adjuvant radiotherapy was recommended for patients with mediastinal lymph node metastasis. The chemotherapy regimen included mainly cisplatin or carboplatin combined with etoposide (platinum and etoposide or carboplatin and etoposide).

\subsection{Statistical methods}

IBM-SPSS 22.0 software was used for data analysis. The Kaplan-Meier survival curve and log-rank univariate test were used to identify the factors correlated with the survival time and prognosis. The logrank test was used in univariate analysis to determine whether a factor had any impact on the survival time. All the variables that showed a statistical significance in univariate analysis were included in multivariate Cox regression analysis, and $P<0.05$ was considered statistically significant.

\section{Results}

\subsection{General characteristics}


During the study period, 136 patients received surgical treatment for SCLC, accounting for $2.1 \%$ $(136 / 6437)$ of the total number of patients who underwent surgical resection for lung tumours in the Department of Thoracic Surgery of PUMCH. After we excluded six patients who had a relatively large amount of data missing, 130 patients were included in the final analysis, including 99 males and 31 females (male:female ratio 3.19:1), with a median age of 60 years (30-83 years) and a median disease duration of 2 months (0.5-48 months). Eighty-seven patients $(66.9 \%)$ had a history of smoking. The ratio of peripheral-type SCLC to central-type SCLC was 2.6:1 (90:34). Nineteen patients (14.6\%) had a definite family history of malignant tumours in their immediate relatives, and seven (5.4\%) had a history of malignant tumours elsewhere before their SCLC diagnosis (Table 1). 
Table 1

Clinical data of 130 SCLC patients

Item

Value $(\mathrm{N}=$

130)

Sex ratio (male:female)

3.2:1 (99:31)

Median age (years)

$60(30-83)$

Median disease duration (months)

$2(0.5-48)$

Smoking/non-smoking

2.0:1 (87:43)

Family history of malignancy

$19(14.6 \%)$

Initial symptoms

$63(48.5 \%)$

Respiratory system (cough and expectoration 33 , bloody sputum 23, chest

$3(2.3 \%)$

tightness 7)

Chest and back pain

$2(1.5 \%)$

Fever

$1(0.8 \%)$

Hyponatraemia

$6(4.6 \%)$

Neurological symptoms* (consciousness disorder 1, limb weakness 5)

$55(42.3 \%)$

Asymptomatic

Peripheral type/central type

$2.6: 1(90 / 34)$

\begin{abstract}
* Six patients had neurological symptoms, including three patients with limb weakness who were clinically diagnosed with paraneoplastic syndrome (Lambert-Eaton syndrome), two patients with nonspecific neurological symptoms, and one patient with consciousness disorder who underwent enhanced MRI to rule out intracranial metastasis and was clinically diagnosed with limbic encephalitis.
\end{abstract}

VATS: video-assisted thoracic surgery

PLT: posterior-lateral thoracotomy

$A B$

$C D$ 


\section{Item}

Value $(\mathrm{N}=$ 130)

Position

$26(20.0 \%)$

The right upper lobe $3(2.3 \%)$

The right $t$ middle lobe $22(16.9 \%)$

The right lower lobe $34(26.2 \%)$

The left upper lobe $32(24.6 \%)$

The left lower lobe

No clear lobulation in the middle of the right lung

No clear lobulation in the middle of the left lung

Surgical approach

$108(83.1 \%)$

VATS

$22(16.9 \%)$

PLT

Scope of surgical resection

$30(23.0 \%)$

Compound lobectomy and above

$90(69.2 \%)$

Lobectomy

$10(7.8 \%)$

Subpulmonary lobectomy

Lymph Nodes management

$117(90.0 \%)$

Lymphadenectomy

Sampling

$2(1.5 \%)$

Biopsy

$6(4.6 \%)$

Not done

The median value of the maximum tumour meridian $(\mathrm{cm})$

$2.9(1.0-12.0)$

* Six patients had neurological symptoms, including three patients with limb weakness who were clinically diagnosed with paraneoplastic syndrome (Lambert-Eaton syndrome), two patients with nonspecific neurological symptoms, and one patient with consciousness disorder who underwent enhanced MRI to rule out intracranial metastasis and was clinically diagnosed with limbic encephalitis.

VATS: video-assisted thoracic surgery

PLT: posterior-lateral thoracotomy

$A B$

C D 


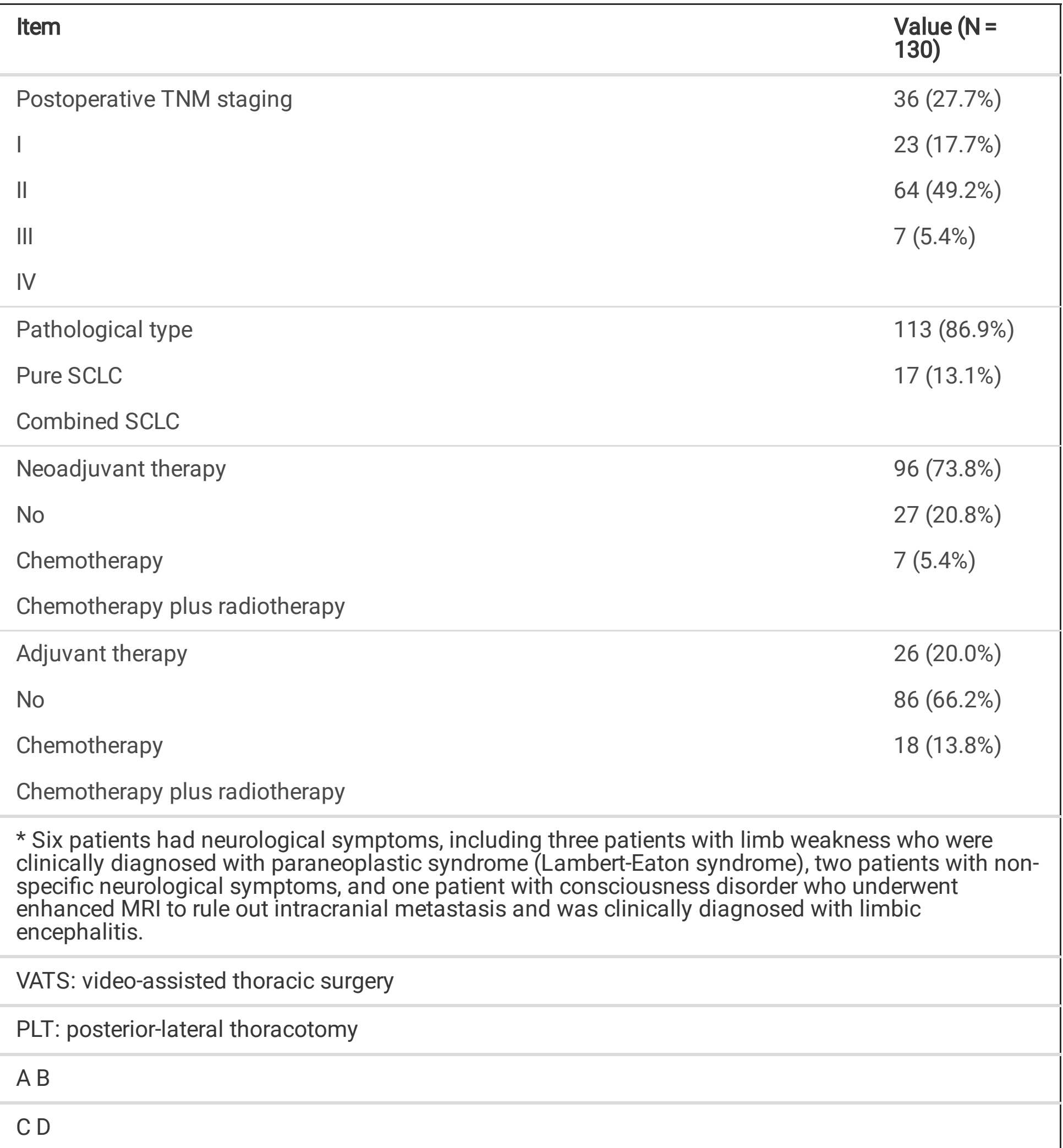

[insert Table 1 here]

\subsection{Clinical symptoms and tumour parameters}

Fifty-five SCLC patients (42.3\%) were asymptomatic and were found during a physical examination. Sixty-three patients (48.5\%) developed respiratory symptoms, including $33(25.4 \%)$ cases of cough and expectoration, $23(17.7 \%)$ cases of coughing up bloody sputum, and 7 (5.4\%) cases of chest congestion. 
In addition, $2(1.5 \%)$ patients had fever, $3(2.3 \%)$ had chest and back pain, and $1(0.8 \%)$ had hyponatraemia. Of the six patients who had neurological symptoms, 5 (3.8\%) presented with limb weakness, and $1(0.8 \%)$ had disorders of consciousness (Table 1$)$. The tumour marker screening revealed that $37(46.8 \%)$ of 79 patients who were tested for neuron-specific enolase had an elevated level (normal reference range $0-16.3 \mathrm{ng} / \mathrm{ml}$ ); among 56 patients who were tested for pro-gastrin-releasing peptide, 46 (82.1\%) had an increase and $28(50 \%)$ had a level exceeding $100 \mathrm{pg} / \mathrm{ml}$ (normal reference range 0$46 \mathrm{pg} / \mathrm{ml})$.

\subsection{Imaging features}

Our patient cohort did not show any specific chest CT features that were meaningful for the differential diagnosis of SCLC from other types of lung cancer. In the peripheral SCLC, the lung lesions mostly appeared as nearly round nodules. The small lesions had relatively smooth boundaries and rarely demonstrated signs of lobulation, burrs, or pleural traction. Such lesions can be easily misdiagnosed as benign lesions in clinical practice (Fig. 1a and b). The large lesions showed slightly enhanced intensity and were lobulated in some cases. The lesions were necrotic inside and seldom showed the signs of burrs or pleura traction (Fig. 1c and d). The tumours could occur in any lung lobe, with the left upper lung being the most common $(34,26.2 \%)$. The median of the longest tumour diameter was $2.9 \mathrm{~cm}(1.0-$ $12.0 \mathrm{~cm}$ ) (Table 1).

\subsection{Preoperative pathological diagnosis}

A total of 55 patients received a preoperative pathological diagnosis, including 45 cases of bronchoscopy biopsy, six cases of CT-guided percutaneous biopsy, three cases of thoracoscopic or mediastinoscopic biopsy, and one case of supraclavicular lymph node biopsy. Among the patients who underwent bronchoscopy biopsy, 30 (66.67\%) were given a definite pathological diagnosis of SCLC. Patients who underwent the latter three procedures were all given a definite pathological diagnosis of SCLC. Among the 15 patients who did not have a definite pathological diagnosis of SCLC via bronchoscopy, 10 had inflammation or necrosis, three had unclassified lung cancer, one had squamous-cell carcinoma, and one had bronchial amyloidosis.

\subsection{Surgery and pathology}

Among all 130 patients, 22 underwent PLT and 108 underwent VATS. Of the VATS group, 9 (8.3\%) were converted to thoracotomy due to hilar lymph node adhesion or invasion (seven patients), pleural adhesion over the whole lung surface (one patient), or intraoperative massive haemorrhage (one patient). Ten patients underwent subpulmonary lobectomy, 90 underwent lobectomy, and 30 underwent compound lobectomy ,the more extensive procedures including lobectomy plus wedge resection, lobectomy plus lobectomy and pneumonectomy. A total of 117 patients underwent lymphadenectomy, five underwent systematic lymph node sampling, two underwent lymph node biopsy, and six did not receive any lymph node management during surgery (Table 1 ). 
Seventy-six patients gave intraoperative rapid frozen pathology specimens, which confirmed SCLC in 51 $(67.1 \%)$ patients. Postoperative pathology diagnosed pure SCLC in 113 patients and combined SCLC in 17 patients, including 10 cases of SCLC having components of adenocarcinoma, four having components of squamous-cell carcinoma, one having components of large-cell neuroendocrine carcinoma (LCNEC), one having components of atypical carcinoid, and one having components of both LCNEC and adenocarcinoma. Two patients (1.5\%) died during the perioperative period: one of postoperative acute myocardial infarction and the other of intraoperative haemorrhage caused by tumour invasion of large blood vessels. Another 14 (10.8\%) patients developed complications during the perioperative period, including two cases of acute coronary syndrome, one case of pulmonary embolism, three cases of nerve injury, two cases of pleural effusion, two cases of arrhythmia, one case of subcutaneous emphysema, one case of lymphatic leakage, one case of epileptic seizure, and one case of poor wound healing. All of the above complications improved, and the patients were discharged from hospital after relevant treatment.

Postoperative pathological staging confirmed stage I SCLC in 36 patients, stage II in 23 patients, stage III in 64 patients, and stage IV in seven patients. Of the seven patients with stage IV SCLC, four had intrathoracic dissemination, one underwent emergency surgery due to tumour rupture and haemorrhage, and two had oligometastasis (the solitary metastatic site was in the liver).

\subsection{Adjuvant therapy and follow-up}

A total of 34 patients received preoperative adjuvant therapy, including 27 who underwent chemotherapy and seven underwent chemotherapy plus radiotherapy. A total of 104 patients underwent postoperative adjuvant therapy, including 86 who underwent chemotherapy and 18 who underwent radiotherapy combined with chemotherapy. The median OS of the 130 patients was 50 months ( $95 \%$ confidence interval, 10.8-89.2 months), and the 3-year, 5-year, and 10-year survival rates were $76.7 \%, 64.0 \%$, and $37.8 \%$, respectively (Fig. 2a). The median survival time of stage I, II, III, and IV SCLC patients was 148 months, 42 months, 32 months, and 10 months, respectively (Fig. 2b). The 5-year survival rate was $90.0 \%$ in stage I patients, $44.0 \%$ in stage II patients, and $25.7 \%$ in stage III patients. Among the seven patients with stage IV SCLC, only one still lived at 14 months after surgery, and the others died of tumour progression (4-19 months after surgery).

\subsection{Survival analysis}

Log-rank univariate analysis showed that the prognosis of SCLC patients was not significantly affected by age, sex, smoking history, tumour position, surgical approach, neoadjuvant chemotherapy, or family history of cancer $(P>0.05)$ (Table 2$)$. The median OS was 21 months $(95 \% \mathrm{Cl}=4.93-37.07), 90$ months $(95 \% \mathrm{Cl}=35.27-144.73)$, and 38 months $(95 \% \mathrm{Cl}=0-106.53)$ in the subpulmonary lobectomy group, the lobectomy group, and the group who underwent compound (or greater) lobectomy. The lobectomy group had a better prognosis than the other two groups (Fig. 4b, $\mathrm{P}=0.048$ ). The lymphadenectomy group had a longer median OS (78 months) than the patients receiving lymph node sampling (19 months), lymph node biopsy (1 months), or no lymph node treatment (21 months) $(P=0.009)$. Patients who received 
adjuvant therapy (chemotherapy, radiotherapy, chemotherapy plus radiotherapy) after surgery had longer OS (Fig. $4 \mathrm{c}, \mathrm{P}=0.019$ ). Tumour stage was also a factor affecting OS (Table 2, $P<0.05$ in both univariate and multivariate analyses). In addition, a comparison of SCLC at different locations revealed that the survival curve showed a certain difference between central and peripheral SCLC, but the difference was not statistically significant (Fig. 3d, $P=0.05$ ). 
Table 2

Univariate log-rank analysis and Cox multivariate analysis of prognostic factors in all 130 SCLC patients after surgical treatment

\begin{tabular}{|c|c|c|c|c|}
\hline \multirow[b]{2}{*}{ Risk factor } & \multirow{2}{*}{$\begin{array}{l}\text { Univariate log- } \\
\text { rank analysis }\end{array}$} & \multicolumn{3}{|c|}{ Multivariate analysis } \\
\hline & & $P$ & HR & $\begin{array}{l}\mathrm{HR} \\
95.0 \% \\
\mathrm{Cl}\end{array}$ \\
\hline Age & 0.957 & & & \\
\hline Sex & 0.234 & & & \\
\hline Smoking history & 0.413 & & & \\
\hline Surgical approach (PLT versus VATS) & 0.569 & & & \\
\hline Neoadjuvant chemotherapy & 0.390 & & & \\
\hline Family history of tumour & 0.670 & & & \\
\hline Tumour location (peripheral type versus central type) & 0.050 & & & \\
\hline $\begin{array}{l}\text { Surgical scope (subpulmonary lobectomy versus } \\
\text { lobectomy and compound lobectomy) }\end{array}$ & 0.015 & 0.760 & 1.256 & $\begin{array}{l}0.291- \\
5.420\end{array}$ \\
\hline Lymphadenectomy (yes versus no) & 0.001 & 0.213 & 2.179 & $\begin{array}{l}0.640- \\
7.421\end{array}$ \\
\hline Adjuvant therapy & 0.019 & 0.014 & 0.374 & $\begin{array}{l}0.171- \\
0.817\end{array}$ \\
\hline Tumour stage & 0.000 & 0.001 & & \\
\hline \multicolumn{5}{|l|}{ Stage I (36) } \\
\hline Stage II (22) & & 0.002 & 5.392 & $\begin{array}{l}1.887- \\
15.405\end{array}$ \\
\hline Stage III (65) & & 0.001 & 4.641 & $\begin{array}{l}1.930- \\
11.158\end{array}$ \\
\hline Stage IV (7) & & 0 & 8.784 & $\begin{array}{l}2.789- \\
27.670\end{array}$ \\
\hline \multicolumn{5}{|c|}{$\begin{array}{l}\text { PLT: posterolateral thoracotomy;VATS: video-assisted thoracic surgery;HR: hazard ratio; Cl:confidence } \\
\text { interval }\end{array}$} \\
\hline \multicolumn{5}{|c|}{$\begin{array}{l}\text { All the above-mentioned variables that had a } P \text { value } \leq 0.05 \text { were introduced into the multivariate Cox } \\
\text { regression analysis. The results suggested that adjuvant therapy }(P=0.014) \text { and tumour-node- } \\
\text { metastasis (TNM) stage }(P=0.01 \text { ) were independent factors affecting prognosis. The higher the } \\
\text { tumour stage, the worse the prognosis: the hazard ratio (HR) was } 5.4 \text { for stage II versus stage I, } 4.6 \text { fol } \\
\text { stage III versus stage II, and } 8.8 \text { for stage IV versus stage III (Table } 2 \text { ). }\end{array}$} \\
\hline
\end{tabular}

\section{Discussion}


For SCLC, the first prospective randomized controlled clinical trial, published in the 1970s, suggested that adjuvant therapy was superior to surgical treatment [6]. Later, a prospective randomized controlled clinical trial conducted in the 1980s also suggested that surgical treatment after adjuvant therapy did not improve the survival of patients with limited-stage SCLC [7]. According to the conclusions from these two clinical trials, chemoradiotherapy-based comprehensive treatment was the optimal option for SCLC for decades. In recent years, with the rapid advancement of preoperative examination technologies and surgical techniques and the advent of a variety of new drugs for chemotherapy, targeting therapy, and immunotherapy, revolutionary progress has been made in the treatment of lung cancer, especially nonsmall-cell lung cancer (NSCLC). However, SCLC, a common lung cancer, still perplexes clinicians, and some researchers even report that no substantial progress has been made in the clinical treatment of SCLC in recent years [8]. Barnes et al. [9] conducted a meta-analysis of recent randomized controlled trials and concluded that surgery has not improved the prognosis of limited-stage SCLC, although they mentioned that the accuracy of the results might be affected by the inconsistences in treatment options and the methods and standards for staging during different times.

Surgical treatment of SCLC remains a hot topic of debate, as the prospective randomized controlled trials that did not support surgical treatment were conducted nearly half a century ago $[6,7]$. At present, the NCCN guidelines recommend surgery followed by adjuvant chemoradiotherapy only to SCLC patients with negative lymph nodes (cT1-2NOM0); for patients with higher-stage SCLC, only comprehensive chemoradiotherapy is recommended. A growing number of clinicians hope to make some new breakthroughs in the surgical treatment of SCLC. Based on data analysis of SCLC patients in the National Cancer Database of the United States from 2003 to 2011, Yang and his colleagues from Duke University found that surgery combined with postoperative chemotherapy improved the prognosis of patients with T1-3 N1M0 SCLC [10]. In addition, a retrospective analysis of SCLC patients from 2004 to 2014 using data from the Surveillance, Epidemiology, and End Results (SEER) database showed that patients with stage I-III SCLC who underwent surgery had a better OS and that those who underwent lobectomy had a better prognosis than those who underwent subpulmonary lobectomy [11]. In the study of Yang et al. [12], surgery brought a survival benefit to patients with stage IIB or stage IIIA SCLC. Zhang et al. [13] suggested that surgery could improve the prognosis of patients with stage III SCLC. Xu et al. [14] found that patients with stage IA, IB, IIA, III, or IV SCLC who received surgical treatment had a significantly prolonged survival time. In addition, the prognosis of patients with stage N0, N1, or N2 SCLC who received surgical treatment was improved [15].

In this study, we retrospectively analysed the single-centre data of 130 SCLC patients who received surgical treatment in PUMCH in the past 15 years (2004-2019). The patients had a median survival time of 50 months and a 5 -year survival rate of $64 \%$. In addition, $49.2 \%$ of the patients enrolled in this study had stage III SCLC, which patients had a median OS of 32 months and a 5 -year survival rate of $25.7 \%$. The results were very encouraging because earlier studies had reported a median OS of only 2-4 months in patients with untreated SCLC, 15-20 months in patients with treated limited-stage SCLC, and 813 months in patients with extended-stage SCLC [3]. Combining our findings with previous ones, we believe that surgery may improve the prognosis of SCLC patients to a certain extent and recommend that 
the surgical indication for SCLC be properly expanded from cT1-2NOMO (recommended by the NCCN guidelines) to stage I-IIla, similar to the surgical indications for NSCLC. The value of surgery in MDT for SCLC needs to be reassessed and verified by more clinical data and randomized controlled trials.

In this study, the patient cohort included mainly elderly male smokers (male:female ratio 3:1, median age 60 years, smoking rate $66.9 \%$ ), which is consistent with most existing SCLC studies. However, more than a half of the patients had no definite clinical symptoms, which is inconsistent with other studies reporting that most patients had clinical symptoms [16]. This might be because the study included only the patients who received surgical treatment; therefore, most of the study subjects had earlier-stage peripheral SCLC. In general, central SCLC is more common, but only a small proportion of central SCLC is resectable due to its invasive growth and early spreading. Bronchoscopic biopsy is the preferred method of pathological diagnosis of SCLC, but a portion of SCLCs with only peripheral lesions are difficult to distinguish from NSCLC based on clinical symptoms and imaging features (Fig. 2), so they are often treated following the diagnosis and treatment procedures of NSCLC. In addition, SCLC lesions are often combined with necrosis and mixed with NSCLC, making a definite diagnosis through small biopsies difficult. In this study, of 45 patients who underwent bronchoscopy before surgery, $66.67 \%$ of them had a definite diagnosis of SCLC, suggesting that pathological diagnosis with small specimens has limitations. Out of the 76 patients who underwent an examination, intraoperative frozen pathology yielded a definite or near-definite diagnosis of SCLC in only $67.1 \%$. As a result, it was difficult to apply preoperative adjuvant therapy, which to a certain extent suggests the necessity of expanding the surgical indications and pathological diagnosis with larger specimens.

The first staging system of SCLC was proposed by the Veterans' Administration Lung Study Group, which divides SCLC into limited stage and extended stage $[17,18]$. The original purpose of this staging method was to distinguish patients whose cancer might be treatable from patients who should receive only palliative treatment. With the advancement of SCLC research, the TNM staging system emerged and has gradually become more widely used. Abraham et al. [19] found that TNM staging was more accurate in predicting distant metastasis, brain metastasis, and survival in limited-stage SCLC patients. Zhu et al. [20] reported that the 3-year incidence of brain metastasis in TNM stage I patients was $9.7 \%$, which was significantly lower than that in TNM stage II (18.5\%) and stage III patients (35.4\%), and they suggested that TNM stage I patients did not need preventive cranial radiation treatment. In this study, the 7th edition of the TNM staging system was used, and tumour stages were included in the survival analysis. The univariate analysis regarding different tumour $(T)$, lymph node $(N)$, and metastasis $(M)$ stages showed that the earlier the stage, the significantly longer the survival time (Fig. 3a-c). The comprehensive univariate analysis of TNM stage also suggested that tumour stage affected the prognosis of SCLC after surgical treatment (Fig. 2b, Table 2). Multivariate analysis confirmed that tumour stage was an independent risk factor affecting the prognosis of SCLC patients after surgical treatment, and the HR gradually increased as the tumour stage increased (Table 2). This suggests that TNM staging can predict the prognosis of SCLC patients and can be used as a basis for formulating treatment plans. 
VATS has become increasingly common. Some $83.1 \%$ of patients enrolled in this study underwent VATS, but there was no difference in prognosis between patients who underwent VATS and those who underwent PLT (Fig. 4a, P = 0.569). Regarding the scope of surgical resection, lobectomy is recommended as the preferred surgical method in the current guidelines. However, various surgical treatment methods, including subpulmonary lobectomy, compound lobectomy, and pneumonectomy, have been used in clinical practice. Given the wish to preserve lung function and the wide application of minimally invasive techniques, subpulmonary lobectomy has been increasingly used for early SCLC, especially in elderly patients ( $>65$ years) and patients with small pulmonary nodules [21]. In the study of Liu et al. [21], the rate of subpulmonary lobectomy in stage I patients reached $31 \%$; neither this study nor previous studies found that subpulmonary lobectomy did not bring a survival benefit to stage I patients [22]. A portion of studies stated that lobectomy led to a more satisfactory prognosis than subpulmonary lobectomy [21, 23, 24], and the prognosis after lobectomy and subpulmonary lobectomy was superior to that after pneumonectomy [25]. In the present study, the patients underwent subpulmonary lobectomy, lobectomy, or compound lobectomy (including pneumonectomy), and the survival curve showed that the prognosis after subpulmonary lobectomy was worse (Fig. $4 b, p=0.048$ ). The univariate analysis suggested that the prognosis of the patients who received lobectomy (or more) was better than that of the patients who received subpulmonary lobectomy (Table $2, P=0.015$ ), but the difference was statistically non-significant in the multivariate analysis. Therefore, we still recommend anatomical lobectomy for patients who can tolerate surgery.

In addition, the impact of lymph node management on the prognosis of SCLC patients after surgery is worth discussing. Chenesseau et al. [26] demonstrated the importance of lymphadenectomy for prognosis. Zhao et al. [27] found that the lymph node metastasis rate was an independent factor affecting prognosis, and stage IIB and IIIA SCLC patients with negative lymph nodes had a better prognosis. Some studies also suggested that in both NSCLC and SCLC, subcarinal lymph node metastasis is an independent factor affecting prognosis $[28,29]$. In our study, based on the pathological characteristics of SCLC and the value of surgical treatment in treating SCLC, four different lymph node management measures were used: Iymphadenectomy, sampling, biopsy, and no treatment. The univariate analysis revealed that lymph node management had an impact on the prognosis (Fig. 4c, $\mathrm{P}=$ 0.009 ) and that the prognosis varied significantly between lymphadenectomy and non-lymphadenectomy (Table 2, $P=0.001)$, though this difference was not significant in the multivariate analysis $(P=0.213)$. However, we still recommend lymphadenectomy because it has potential therapeutic value and can provide more accurate $\mathrm{N}$ staging.

Adjuvant chemoradiotherapy plays a particularly important role in the surgery-centred treatment for SCLC. The current NCCN guidelines recommend adjuvant chemoradiotherapy for SCLC patients after surgery and suggest that the addition of radiotherapy be determined based on the status of mediastinal lymph node metastasis. However, the significance of adjuvant chemoradiotherapy after surgery for different stages of SCLC patients remains controversial. Luchtenborg et al. [30] and Wong et al. [31] believed that postoperative adjuvant chemoradiotherapy could reduce the local recurrence rate of SCLC. Two recent studies that included the data of surgically treated SCLC patients in the SEER database from 2010 to 
2015 and from 2004 to 2015, respectively, found that postoperative adjuvant chemotherapy and radiotherapy improved the prognosis of patients $[14,32]$. However, another single-centre retrospective study involving 205 surgically treated SCLC patients found that postoperative adjuvant radiotherapy did not improve the prognosis of stage I-III SCLC patients [27]. Engelhardt et al. [33] also found that SCLC patients did not benefit from postoperative adjuvant radiotherapy. Our study suggested that postoperative adjuvant therapy (chemotherapy, radiotherapy, or chemoradiotherapy) may have survival benefits (Fig. 4d, Table 2, univariate survival analysis $P=0.019$, multivariate analysis $P=0.014$ ). Zhong et al. [34] reported that preoperative neoadjuvant radiochemotherapy did not improve the prognosis of patients who were surgically treated for SCLC, and our study did not find any prognostic benefit of neoadjuvant therapy $(P=0.39)$. However, because this study is a retrospective study with a long time span, the chemotherapy and radiotherapy regimens and the implementation standards of neoadjuvant therapy for patients were inconsistent throughout the study period. Therefore, stratified analyses of preoperative adjuvant therapy, postoperative chemotherapy alone, postoperative radiotherapy alone, and postoperative combined chemoradiotherapy for SCLC need more data or prospective studies.

We also noticed a difference in survival between central and peripheral SCLC patients. The univariate analysis showed that the median OS of central SCLC patients (58 months) tended to be longer than that of the peripheral SCLC (30.5 months), but the difference was not statistically significant (Fig. 3d, Table 2, $P=0.05$; multivariate analysis $P=0.078$ ). In a study published by Woo et al. [35] in 2019, the prognosis of central SCLC patients was worse than that of peripheral SCLC patients after surgery. Only 10 patients with central SCLC were included in that study, and they had the same mean OS and disease-free survival (DFS) of 44.6 months, which was worse than in the peripheral SCLC patients (179.2 months and 145.3 months, respectively) ( $P=0.017, P=0.233$, respectively) [35]. The 2017 study by Kanaji et al. [36] found that the prognosis of central SCLC patients without surgical treatment was worse than that of peripheral SCLC patients without surgical treatment. Peripheral SCLC patients in the present study were more likely to accept subpulmonary lobectomy, especially wedge resection, and they did not undergo further anatomical lobectomy or lymphadenectomy, following to the existing SCLC treatment strategy when SCLC was confirmed from specimens harvested by wedge resection during surgery. This might be the reason behind the relatively poor outcome of peripheral SCLC in this study.

Our research has some limitations. This was a retrospective single-centre study with a long time span, and as a result, the treatment options for SCLC were inconsistent during the study period. Our findings need to be verified by a multi-centre study with a large sample and by prospective clinical trials.

In summary, using relevant SCLC surgical treatment data in a single centre (PUMCH), the present retrospective study concludes that surgery combined with adjuvant therapy can bring a survival benefit to selected SCLC patients; tumour stage and postoperative adjuvant therapy are independent risk factors affecting the prognosis. We recommend that the surgical indications for SCLC be extended to preoperatively diagnosed stage I-IIla tumours and that treatment include anatomical lobectomy and lymphadenectomy combined with postoperative adjuvant therapy. 


\section{Abbreviations}

MDT multi-disciplinary treatment

OS overall survival

PUMCH Peking Union Medical College Hospital

PLT posterolateral thoracotomy

VATS video-assisted thoracic surgery

LCNEC large-cell neuroendocrine carcinoma

SEER Surveillance, Epidemiology, and End Results

\section{Declarations}

Ethics approval and consent to participate

Approved by the institutional review board(IRB) of PUMCH, Protocal Number S-K1227

Consent for publication

yes

\section{Availability of data and materials}

The datasets during and/or analysed during the current study available from the corresponding author on reasonable request.

\section{Competing interests}

none

\section{Funding}

no

\section{Authors' contributions}

CYY participated in research design, collected and analyzed the data, and was a major contributor in writing the manuscript. LL participated in data collection and was a major contributor in statistical analysis. $\mathrm{HC}$ participated in data analyzation and chart arrangement. TZH participated in data collection and patients follow up. LHS participated in research design. LSQ participated in research design, and was 
a major contributor in monitoring the research process and was responsible for the analysis and interpretation of the results.

\section{Acknowledgements}

\section{Authors' information}

\section{References}

1. Kahnert K, Kauffmann-Guerrero D, Huber RM. SCLC-state of the art and what does the future have in store? Clin Lung Cancer. 2016;17:325-33.

2. Kato Y, Ferguson TB, Bennett DE, Burford TH. Oat cell carcinoma of the lung. A review of 138 cases. Cancer. 1969;23:517-24.

3. van Meerbeeck JP, Fennell DA, de Ruysscher DK. Small-cell lung cancer. Lancet. 2011;378:1741-55.

4. National Comprehensive Cancer Network. NCCN clinical practice guidelines in oncology: small cell lung cancer (version 2.2015). Fort Washington: National Comprehensive Cancer Network; 2015.

5. Lababede O, Meziane M, Rice T. Seventh edition of the cancer staging manual and stage grouping of lung cancer: quick reference chart and diagrams. Chest. 2011;139:183-9.

6. Fox W, Scadding JG. Medical research council comparative trial of surgery and radiotherapy for primary treatment of small-celled or oat-celled carcinoma of bronchus. Ten-year follow-up. Lancet. 1973;2:63-5.

7. Lad T, Piantadosi S, Thomas P, Payne D, Ruckdeschel J, Giaccone G. A prospective randomized trial to determine the benefit of surgical resection of residual disease following response of small cell lung cancer to combination chemotherapy. Chest. 1994;106:320S-3.

8. Koinis F, Kotsakis A, Georgoulias V. Small cell lung cancer (SCLC): no treatment advances in recent years. Transl Lung Cancer Res. 2016;5:39-50.

9. Barnes H, See K, Barnett S, Manser R. Surgery for limited-stage small-cell lung cancer. Cochrane Database Syst Rev. 2017;4:CD011917.

10. Yang CJ, Chan DY, Speicher PJ, Gulack BC, Tong BC, Hartwig MG, et al. Surgery versus optimal medical management for N1 small cell lung cancer. Ann Thorac Surg. 2017;103:1767-72.

11. Che K, Shen H, Qu X, Pang Z, Jiang Y, Liu S, et al. Survival outcomes for patients with surgical and non-surgical treatments in stages I-III small-cell lung cancer. J Cancer. 2018;9:1421-9.

12. Yang $Y$, Yuan G, Zhan $C$, Huang $Y$, Zhao M, Yang $X$, et al. Benefits of surgery in the multimodality treatment of stage IIB-IIIC small cell lung cancer. J Cancer. 2019;10:5404-12.

13. Zhang C, Li C, Shang X, Lin J, Wang H. Surgery as a potential treatment option for patients with stage III small-cell lung cancer: a propensity score matching analysis. Front Oncol. 2019;9:1339. 
14. Xu L, Zhang G, Song S, Zheng Z. Surgery for small cell lung cancer: a surveillance, epidemiology, and end results (SEER) survey from 2010 to 2015. Med (Baltim). 2019;98:e17214.

15. Schreiber D, Rineer J, Weedon J, Vongtama D, Wortham A, Kim A, et al. Survival outcomes with the use of surgery in limited-stage small cell lung cancer: should its role be re-evaluated? Cancer. 2010;116:1350-7.

16. Kalemkerian GP. Small cell lung cancer. Semin Respir Crit Care Med. 2016;37:783-96.

17. Rami-Porta R, Bolejack V, Giroux DJ, Chansky K, Crowley J, Asamura H, et al. The IASLC lung cancer staging project: the new database to inform the eighth edition of the TNM classification of lung cancer. J Thorac Oncol. 2014;9:1618-24.

18. Micke P, Faldum A, Metz T, Beeh KM, Bittinger F, Hengstler JG, et al. Staging small cell lung cancer: veterans administration lung study group versus international association for the study of lung cancer-what limits limited disease? Lung Cancer. 2002;37:271-6.

19. Wu AJ, Gillis A, Foster A, Woo K, Zhang Z, Gelblum DY, et al. Patterns of failure in limited-stage small cell lung cancer: implications of TNM stage for prophylactic cranial irradiation. Radiother Oncol. 2017;125:130-5.

20. Zhu H, Bi Y, Han A, Luo J, Li M, Shi F, et al. Risk factors for brain metastases in completely resected small cell lung cancer: a retrospective study to identify patients most likely to benefit from prophylactic cranial irradiation. Radiat Oncol. 2014;9:216.

21. Liu Y, Shan L, Shen J, Liu L, Wang J, He J, et al. Choice of surgical procedure - lobectomy, segmentectomy, or wedge resection - for patients with stage T1-2NOM0 small cell lung cancer: a population-based study. Thorac Cancer. 2019;10:593-600.

22. Varlotto JM, Recht A, Flickinger JC, Medford-Davis LN, Dyer AM, DeCamp MM. Lobectomy leads to optimal survival in early-stage small cell lung cancer: a retrospective analysis. $J$ Thorac Cardiovasc Surg. 2011;142:538-46.

23. Yang $\mathrm{H}, \mathrm{Xu} \mathrm{J}$, Yao F, Liang $\mathrm{S}$, Zhao H. Analysis of unexpected small cell lung cancer following surgery as the primary treatment. J Cancer Res Clin Oncol. 2018;144:2441-7.

24. Paximadis P, Beebe-Dimmer JL, George J, Schwartz AG, Wozniak A, Gadgeel S. Comparing treatment strategies for stage I small-cell lung cancer. Clin Lung Cancer. 2018;19:e559-65.

25. Lohinai Z, Bonanno L, Aksarin A, Pavan A, Megyesfalvi Z, Santa B, et al. Neutrophil-lymphocyte ratio is prognostic in early stage resected small-cell lung cancer. PeerJ. 2019;7:e7232.

26. Chenesseau J, Bourlard D, Cluzel A, Trousse D, D'Journo XB, Thomas PA. Intent-to-cure surgery for small-cell lung cancer in the era of contemporary screening and staging methods. Interact Cardiovasc Thorac Surg. 2020;30:541-5.

27. Zhao X, Kallakury B, Chahine JJ, Hartmann D, Zhang Y, Chen Y, et al. Surgical resection of SCLC: prognostic factors and the tumor microenvironment. J Thorac Oncol. 2019;14:914-23.

28. Okada M, Tsubota N, Yoshimura M, Miyamoto Y, Matsuoka H. Prognosis of completely resected pN2 non-small cell lung carcinomas: what is the significant node that affects survival? J Thorac Cardiovasc Surg. 1999;118:270-5. 
29. Miyamoto M, Morikawa T, Kaga K, Ohtake S, Cho Y, Hirano S, et al. Subcarinal node is the significant node that affects survival in resected small cell lung cancer. Surg Today. 2006;36:671-5.

30. Luchtenborg M, Riaz SP, Lim E, Page R, Baldwin DR, Jakobsen E, et al. Survival of patients with small cell lung cancer undergoing lung resection in England, 1998-2009. Thorax. 2014;69:269-73.

31. Wong AT, Rineer J, Schwartz D, Schreiber D. Assessing the impact of postoperative radiation therapy for completely resected limited-stage small cell lung cancer using the national cancer database. $J$ Thorac Oncol. 2016;11:242-8.

32. Peng A, Li G, Xiong M, Xie S, Wang C. Role of surgery in patients with early stage small-cell lung cancer. Cancer Manag Res. 2019;11:7089-101.

33. Engelhardt KE, Coughlin JM, DeCamp MM, Denlinger CE, Meyerson SL, Bharat A, et al. Survival after adjuvant radiation therapy in localized small cell lung cancer treated with complete resection. $J$ Thorac Cardiovasc Surg. 2019;158:1665-77.e2.

34. Zhong L, Suo J, Wang Y, Han J, Zhou H, Wei H, et al. Prognosis of limited-stage small cell lung cancer with comprehensive treatment including radical resection. World J Surg Oncol. 2020;18:27.

35. Woo JH, Kim MY, Lee KS, Jeong DY, Chung MJ, Han J, et al. Resected pure small cell lung carcinomas and combined small cell lung carcinomas: histopathology features, imaging features, and prognoses. AJR Am J Roentgenol. 2019;212:773-81.

36. Kanaji N, Sakai K, Ueda Y, Miyawaki H, Ishii T, Watanabe N, et al. Peripheral-type small cell lung cancer is associated with better survival and higher frequency of interstitial lung disease. Lung Cancer. 2017;108:126-33.

\section{Figures}




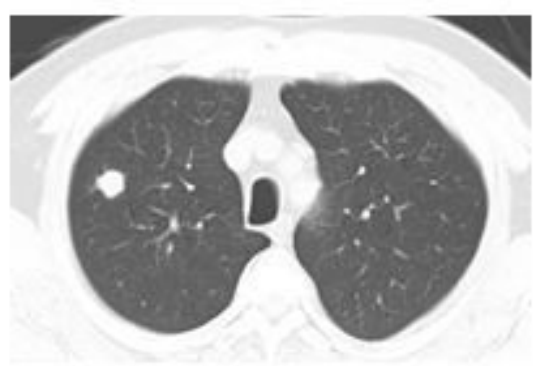

A

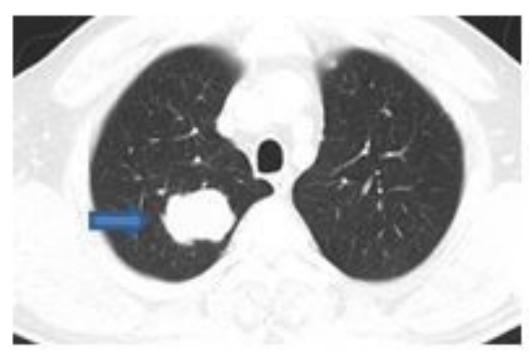

$\mathrm{C}$

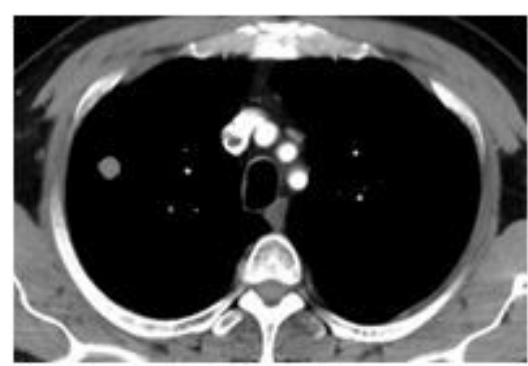

B

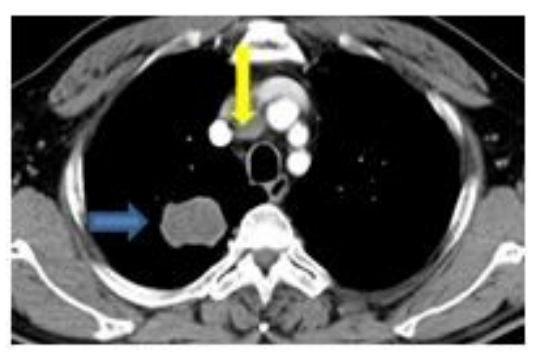

D

Figure 1

Representative CT images of SCLC patients included in this study a and b: A case of peripheral-type SCLC: The lesion was manifested by a nearly round nodule with a relatively smooth boundary and no obvious signs of lobulation, burrs, or pleural traction. $c$ and d: Another case of peripheral-type SCLC: The lesion showed superficial lobulation and did not have obvious burrs or pleural traction; it was slightly enhanced, showing some low-density dark necrotic areas.

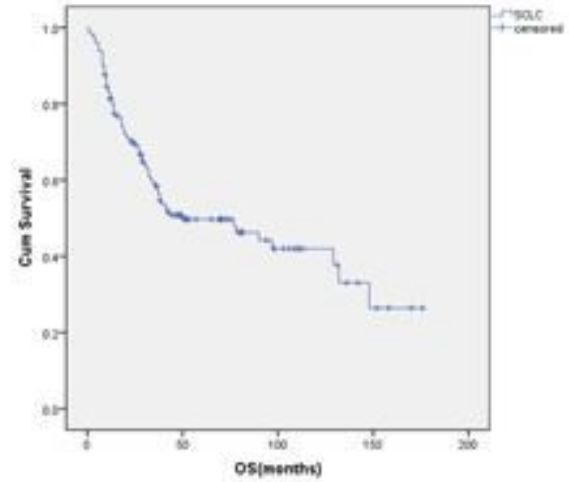

A

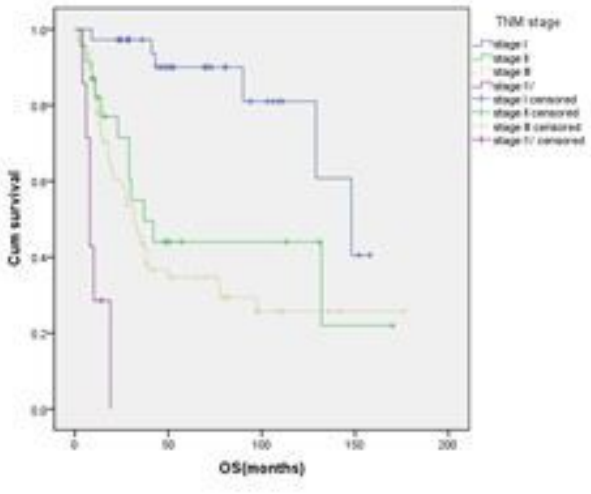

B

\section{Figure 2}

a. OS curve of patients in this study. The median OS was 50.0 months ( $95 \% \mathrm{Cl} 10.8-89.2$ months). The 3-, 5 -, and 10 -year survival rates were $76.7 \%, 64.0 \%$, and $37.8 \%$, respectively. b. Survival curves of patients 
with different stages of SCLC. The median survival time was 148 months for stage I, 42 months for stage II, 32 months for stage III, and 10 months for stage IV.
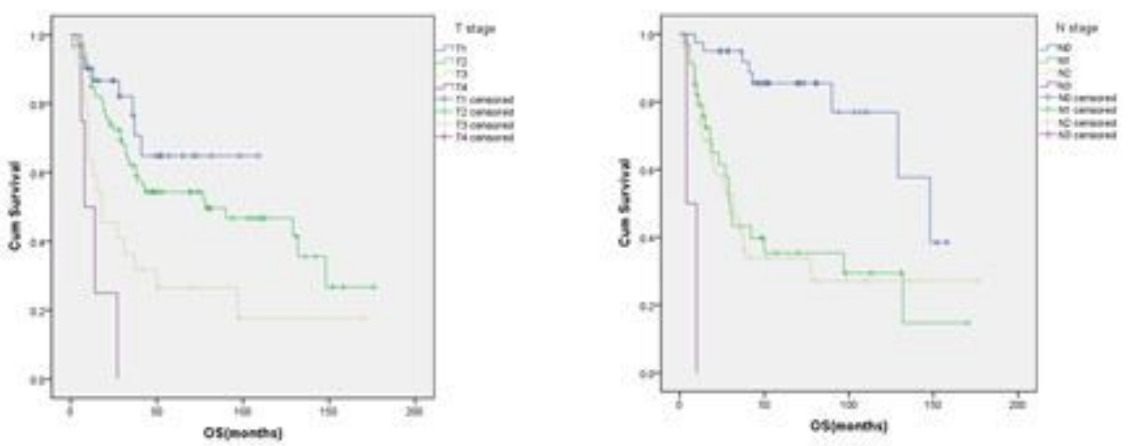

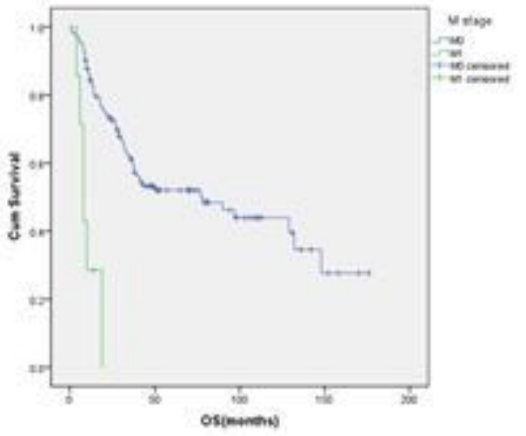

A

$\mathrm{C}$

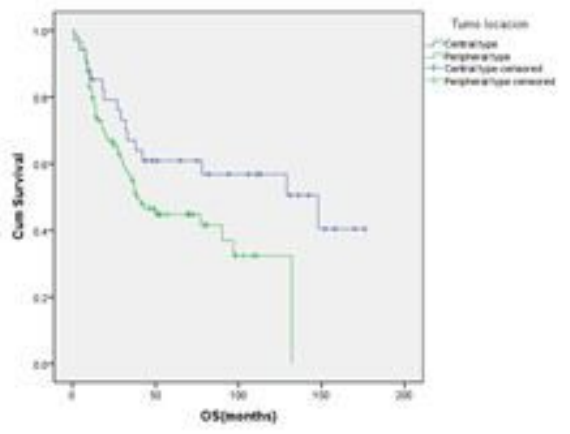

B

D

\section{Figure 3}

The survival curves of patients with SCLC in different T stages (a), N stages (b), and M stages (c) and at different locations (peripheral type and central type) (d). Univariate log-rank analysis: T stage $\mathrm{P}=0.00, \mathrm{~N}$ stage $P=0.00, M$ stage $P=0.00$, and tumour location $P=0.50$. 


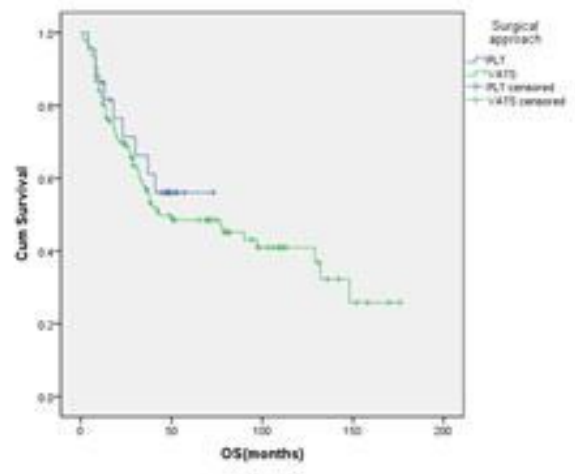

A

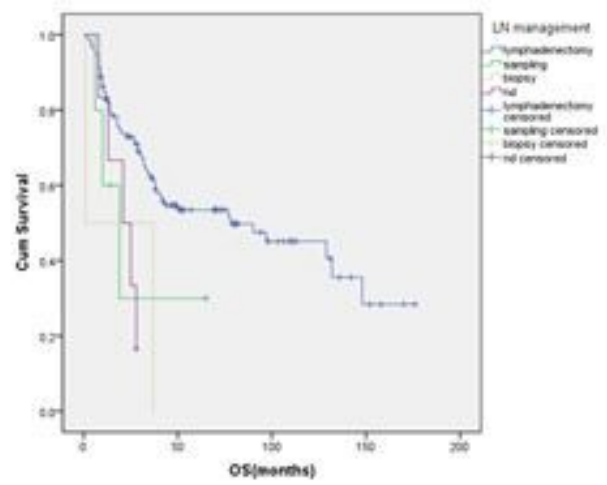

C

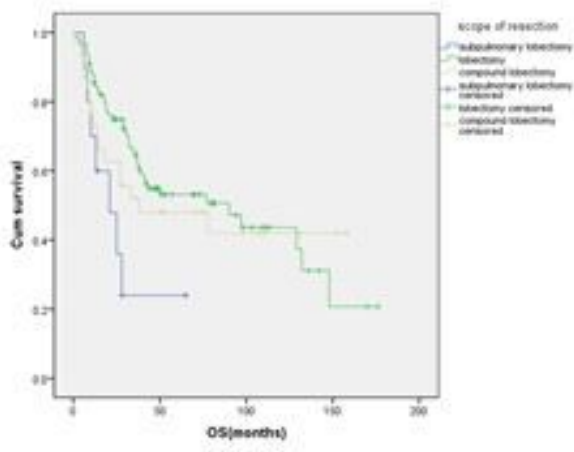

B

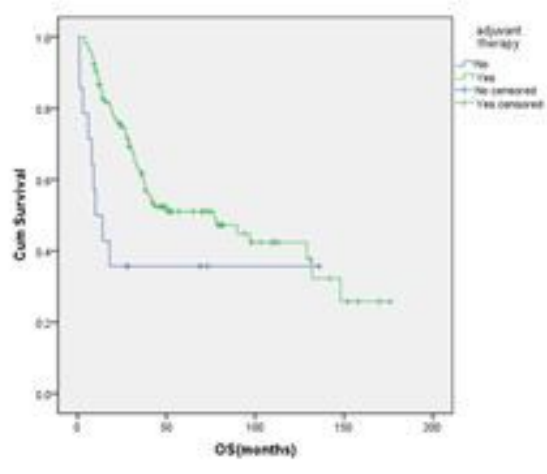

D

\section{Figure 4}

Survival curves of patients who received different surgical approaches and adjuvant therapy. a. Surgical approach $(P=0.569)$. b. Scope of resection $(p=0.048)$. c. Lymph node management $(P=0.009)$. $d$. Adjuvant therapy $(P=0.019)$. PLT: posterolateral thoracotomy VATS: video-assisted thoracic surgery 